\title{
Is routine growth monitoring effective? A systematic review of trials
}

\author{
Paul Garner, Ratana Panpanich, Stuart Logan
}

\begin{abstract}
Background-Growth monitoring consists of routine measurements to detect abnormal growth, combined with some action when this is detected. It aims to improve nutrition, reduce the risk of death or inadequate nutrition, help educate carers, and lead to early referral for conditions manifest by growth disorders. As primary care workers world wide invest time in this activity, evidence for its benefits and harms was sort.

Inclusion criteria-Studies: randomised or quasi-randomised controlled trials of growth monitoring. Interventions: regular growth monitoring, combined with some intervention targeted at abnormal growth, compared with controls. Outcomes: anthropometric measures; referrals to primary and specialist care, or community services; maternal knowledge, anxiety, and satisfaction; child morbidity and mortality. Comparisons-Routine growth monitoring compared with no routine growth monitoring; routine growth monitoring by plotting onto a standard chart compared with monitoring with no chart.
\end{abstract}

Search strategy-Cochrane controlled trials register; World Health Organisation and World Bank publications; contact with specialist community paediatricians working in the field.

Results-Two trials met the inclusion criteria. One compared growth monitoring with no growth monitoring, in a cluster randomised trial nested in a nutritional intervention programme, and detected no difference in nutritional outcomes between the two groups. Another trial compared growth monitoring with and without a standard chart, measuring maternal knowledge of women about nutrition. It showed small numerical differences in test scores.

Discussion and implications-Current policies appear to be based on the opinion that investment in the activity has worthwhile health benefits, and does no harm. No reliable evidence was found to support or refute this.

(Arch Dis Child 2000;82:197-201)

Keywords: growth monitoring; systematic review

Health professionals accept routine growth monitoring in children as a standard component of community child health services throughout the world. ${ }^{1}$ All programmes aim at detecting abnormal growth, and this is fol-
Box 1 What is growth monitoring?

- Regularly measuring the weight (or height) of children

- Plotting the information on a growth chart to make abnormal growth visible

- Where growth is abnormal, the health worker does something, in concert with the family

- As a result of these actions, the child's nutrition improves, the child receives appropriate social or medical support; or, after appropriate investigation, a serious condition is diagnosed earlier

lowed by nutritional or social intervention, or further investigation for serious diseases (box 1). However, the purpose, objectives, and indicators of achievement of growth monitoring programmes are rarely made explicit, and vary. ${ }^{2}$ We therefore first explored growth monitoring in relation to its setting, objectives, and outcomes relevant to the objectives.

\section{SETTING}

In developing countries, health workers monitor growth to detect and intervene in children with evidence of malnutrition. Health workers and mothers spend time on this activity. ${ }^{3}$ The World Bank promote it, claiming high impact for some intensively supervised development projects. ${ }^{2}$ They cite, for example, the Iringa project in Tanzania, where growth monitoring mobilises communities in complex programmes aimed at improving nutrition. ${ }^{4} \mathrm{~A}$ narrative overview of 10 such projects suggested that growth monitoring (used in three of them) has the potential for "a significant impact on mortality . . .even in the absence of nutrition supplementation or education". ${ }^{5}$ The assumption that growth monitoring is of benefit has meant that most research has been directed at improving accuracy of measurement and interpretation, in both developing ${ }^{6} 7$ and developed countries. ${ }^{8}$

In developed countries, growth faltering is seen as an indicator of other social or health problems requiring appropriate intervention. This may lead to social interventions, or further medical investigation for conditions such as failure to thrive (box 2). Particular concerns for specialist paediatricians are when is it best to ensure all children are screened to detect abnormalities; and when to measure children routinely as a method for detecting social deprivation and failure to thrive. The focus of this review is programmes that 


\section{Box 2 Common causes of growth faltering, and responses to these problems}

COMMON CAUSES

- Feeding difficulties, particularly in the younger child

- Chronic ill health from whatever cause, including respiratory infection, malaria, tuberculosis

- Social deprivation, where poverty and home circumstances are such that one outcome is poor nutrition

- "Non-organic failure to thrive", a broader term with multiple associated causes

- Child abuse

COMMON INTERVENTIONNS

- Counselling of the mother

- Counselling of the mother aided by the growth chart

- Nutritional supplement

- Treatment of concurrent disease, such as diarrhoea

- Investigation for disease by the practitioner

- Referral to a multidisciplinary team for investigation and diagnosis

- Professional health worker or social support

regularly monitor growth. We therefore excluded single population measures, such as height at one or more specific ages, which aim to screen for conditions such as growth hormone deficiency.

\section{OUTCOMES}

Definition of purpose is required to evaluate growth monitoring. For example, in developing countries, growth monitoring often aims at detecting and intervening in malnutrition thereby reducing the risk of death; thus it can be evaluated by measuring its impact on nutritional status and death rates. Sometimes growth monitoring is used as part of health promotion, to discuss feeding, hygiene, and other aspects of the child's health and behaviour ${ }^{1}$; maternal nutritional knowledge is a relevant outcome when this is the purpose. Some health workers use growth monitoring to reassure parents about their child's growth, and convince them of the value of good nutrition. These benefits are less easily measured, but might be evaluated by examining parental satisfaction with the services. Such an evaluation should also include parental satisfaction when abnormal growth is detected in their child.

Growth monitoring can potentially cause harm. Parents might become anxious about their child's weight, and may feel guilty if the clinic detects poor weight gain or loss of weight between visits. If the health worker appears to blame the mother, she may be reluctant to return to the clinic, and miss interventions known to be of benefit, such as vaccines. Evaluation should therefore measure these outcomes too.

\section{QUESTIONS}

Our systematic review sought to answer the following questions. Does routine growth monitoring in children:

- Prevent death, illness or malnutrition?

- Increase appropriate referrals for medical care, specialist assessment, or social interventions?

- Improve the nutritional knowledge of the carers?

- Create anxiety in the carers, or reassure them?

\section{Inclusion criteria}

We sought randomised or quasi-randomised comparisons of growth monitoring programmes in children. Given the number of co-interventions associated with growth monitoring and the potential for confounding, we excluded observational studies.

The interventions we sought were regular monitoring (at least three monthly) of growth through measurement of weight, height, or mid upper arm circumference; conversion of these measures to weight for age, height for age, and/or weight for height; combined with some intervention targeted at those with faltering growth. Children attending regular child clinics, or children offered no regular clinics served as controls. Trials in any setting were sought, including refugee camps.

The outcomes we sought were:

- Anthropometric indicators of nutrition

- Responses to illnesses, including referrals to primary care and to specialist services

- Referrals to community services

- Maternal outcomes expressed as knowledge, anxiety, and satisfaction

- Child morbidity and mortality outcomes.

\section{Search strategy}

We searched the Cochrane controlled trials register, published by the Cochrane Library, last searched disk issue 2, 1999. This contains all trials found by handsearching over 1000 journals, including regional journals from developing countries. ${ }^{9}$ We also searched MEDLINE 1966-98; EMBASE 1988-98; CINAHL 1993-98, using the following subject and textword terms: anthropometry; child; child growth; child health; community health; failure to thrive; growth; growth chart; growth monitoring; health visitor; height; infant; monitoring; nutrition; thrive; weight. Organisations and individual researchers working in the field were contacted for unpublished data, confidential reports, and raw data of published trials. External referees were asked to check the completeness of the search strategy and the efforts made to identify trials.

\section{Data analysis}

Two people independently applied inclusion criteria and extracted data. We formed two comparisons: routine growth monitoring 
compared with no routine growth monitoring; and routine growth monitoring by plotting on to a standard chart, compared with monitoring with no chart.

\section{Description of studies}

Ten possible studies were identified. Eight did not meet the entry criteria: one compared interventions in children not thriving, with growth monitoring in both groups ${ }^{10}$; another used growth monitoring to identify children who were then recruited into the study. ${ }^{11}$ One compared early discharge of low birth weight infants with hospitalisation ${ }^{12}$; and others were various observational studies. ${ }^{13}$ None of the studies identified in the search tested height measures, and none tested whether nutritional variables measured routinely one to four times during childhood were of benefit. The two trials that met the inclusion criteria were conducted in developing countries. ${ }^{14}{ }^{15}$ Ruel et al published their results in four different journals, and we used the data from the first published article. ${ }^{15}$

One study from India randomly allocated six pairs of villages to receive or not to receive routine growth monitoring. ${ }^{14}$ The study was nested in a health intervention programme, with both groups receiving a nutritional and healthcare package at weekly rural clinics. In the growth monitoring villages a community health worker weighed the children every month and followed a specific protocol of responses if growth faltered. ${ }^{16}$ No routine weighing was conducted in the control villages. Mean weight gain and mean height gain in the children were then compared over the 30 months' period of intervention through five cross sectional surveys. The method of allocating groups in the six pairs was not clear and there was no blinding. Follow up was good, but there were some differences in feeding practices between the two groups.

One study in Lesotho studied nine primary healthcare clinics over four months. ${ }^{15}$ Mothers were assigned sequentially to the growth surveillance chart (367), road to health chart (389), or no chart (465). At each monthly clinic, mothers were "taught how to interpret the respective growth chart", emphasising direction of growth. The control group received no instruction about the chart. On the fourth and final visit, mothers were tested for their knowledge about the growth chart, weaning practices, and nutrition management; $335 / 1221(27 \%)$ mothers did not complete the four visits.

\section{Results}

In the Indian study mean $\mathrm{Z}$ scores of weight for age and height for age were similar in the villages both with and without growth monitoring. Scores improved in both intervention and control arms, ${ }^{14}$ and no difference in mean weight and height gain during the 30 months was detected. The weight gains in the good growing season were twice those in the poor growing season.

In the Lesotho study the authors used a particular growth chart combined with teaching mothers how to interpret growth curves. This improved the mean scores of tests for maternal comprehension of growth patterns, over an implementation phase of four months. The authors reported that the greatest percentage score improvement adjusted for baseline score was in the road to health chart, though the significance of this is unclear.

No study evaluated the effects of growth monitoring on child morbidity or mortality; or measured maternal satisfaction or anxiety in relation to results of health worker feedback at the time of weighing. No studies were in developed countries, and no studies evaluated referral as an outcome.

\section{Discussion}

The Indian study showed some baseline differences in the intervention and control groups, and the overall nutrition intervention appeared to benefit both groups. The authors were fairly certain that the growth monitoring was properly conducted by staff, and that specific advice was given when growth faltered, so the absence of effect is unlikely to be due to failed implementation. The trial was cluster randomised by village, thereby increasing the amount of statistical uncertainty around any estimate of effects calculated. Thus the absence of an impact on nutritional status may reflect a true absence of impact, or the result of the study being too small to detect a difference between the two groups.

The Lesotho study suggests some differences in maternal interpretation of growth charts between women instructed in their use and women who had not been so instructed. The knowledge evaluation was done at the same time as the final clinic nutrition counselling session, and thus the results may simply reflect short term recall of information presented to them earlier that day. Using a scoring system to test knowledge and then presenting means makes it difficult for the reader to understand exactly what the score actually reflects.

\section{Implications for practice}

At present, it is our opinion that there is insufficient reliable information to be confident about whether routine growth monitoring is of benefit to child health in either developing or developed countries. Thus it is not clear to us whether health professionals should actively pursue children to obtain measures of growth at arbitrarily defined intervals. This includes home visits for children who have not attended clinics at predefined ages.

Growth monitoring is an intrinsic part of "well baby" checks in many parts of the world. It is important that future work seeks to clarify whether any benefits accruing from routine checks are a result of the growth monitoring or other aspects of attending for routine checks. It seems unlikely that in the developed world health professionals will stop weighing babies. The purpose of such weighing, clear criteria for action, and the appropriate course of action should be clearly spelt out if we are to avoid the risk of doing more harm than good. 


\section{Implications for research}

Growth monitoring is routine in many settings but it has been inadequately evaluated, possibly because intuitively it does not appear harmful. However, this assumption may be incorrect as growth monitoring can cause anxiety in the carer (usually the mother) and large amounts of valuable health worker and carer time are expended in carrying it out. A trial testing growth monitoring will depend on the setting and the primary purpose of the monitoring. Researchers will need to:

- Define clearly the intervention, including the actions that arise out of detecting faltering growth

- Randomise participants (by clinic or village), and ensure sufficient data are collected to correct for design effects

- Identify and define outcomes carefully, dependent on the agreed purpose of the growth monitoring. These should include nutritional status. They may include measures of medical referral, treatments given or conditions detected; information about maternal knowledge, feeding practices, and satisfaction with the service

- Include representatives of parents in the planning of the study, to help particularly with defining parental satisfaction outcomes.

This review was developed as an activity of the Effective Health Care in Developing Countries Project, supported by a grant from the Department for International Development, UK and the European Union DG XII. The funders take no responsibility for the data presented or the views expressed. We are grateful to Mohga Smith who commented on an earlier version of the review.

This review appears in the Cochrane Library, and will be updated as new trials emerge.

1 Hall DMB. Health for all children. Report of the third joint working party on child health surveillance. 3 rd ed (reprinted with corrections). Oxford: Oxford University Press, 1996.

2 Griffiths M, Dickin K, Favin M. Promoting the growth of Griffiths M, Dickin K, Favin M. Promoting the growth of
children: what works. Rationale and guidance for programs. children: what works. Rationale and guidance for programs. 1996.

3 Reid J. The role of maternal and child health clinics in education and prevention: a case study from Papua New Guinea. Soc Sci Med 1984;19:291-30.

4 Jonsson U, Ljungqvist B, Yambi O. Mobilization for nutrition in Tanzania. In: Rohde J, Chatterjee M, Morley $\mathrm{D}$, eds. Researching health for all. Oxford: Oxford University Press, 1993.

5 Gwatkin DR, Wilcox JR, Wray JD. Can health and nutrition interventions make a difference? Washington DC: Overseas Development Council. (Monograph No 13.)

6 Meegan M, Morley DC, Brown R. Child weighing by the unschooled: a report of a controlled study of growth moni-
toring over 12 months of Maasai children using direct toring over 12 months of Maasai children using direct

7 Senanayake MP, Gunawardena MKS, Peiris DSP. Maternal comprehension of two growth monitoring charts in Sri comprehension of two growth monito
Lanka. Arch Dis Child 1997;76:359-61.

8 Davies DP, Williams T. Is weighing babies in clinics worthDavies DP, Williams T. Is weig
while? BMF 1983;286:860-3.

9 New England Cochrane Center. Master list of journals being searched. On: http://www.cochrane.org/srch.htm

10 Black MM, Dubowitz H, Hutcheson J, et al. A randomized trial of home intervention for children with failure to thrive. Pediatrics 1995;95:807-14

11 Wright CM, Callum J, Birks E, et al. Effect of community based management in failure to thrive: randomised controlled trial. BMF 1998;317:571-4.

12 Casiro OG, McKenzie ME, McFayden L, et al. Earlier discharge with community-based intervention for low birth weight infants: a randomized trial. Pediatrics 1993;92:12834.

13 Panpanich R, Garner P. Growth monitoring in children. Cochrane Review 1999: issue 2. (Oxford: Update software.) 4 George SM, Latham MC, Abel R, et al. Evaluation of effectiveness of good growth monitoring in South Indian tiveness of good growth moni
villages. Lancet 1993;342:348-52.

15 Ruel MT, Pelletier DL, Habicht JP, et al. Comparison of mothers understanding of two child growth charts in Lesotho. Bull WHO 1990;68:483-91.
16 George SM. The effectiveness of growth monitoring in South Indian villages. PhD dissertation. New York: Cornell Indian villages.
University, 1993.

\section{Commentary}

Growth monitoring of infants and young children is accepted as one of the universal pillars of primary child health care. Less than satisfactory growth suggests all is not well with the health of the young infant, which, with appropriate remedial action, can hopefully be restored. Growth monitoring in day to day clinical practice in less developed countries places special emphasis on weight gain; in the more developed world growth monitoring commonly involves additional parameters, notably head circumference and length. In this commentary weight changes and growth monitoring are used synonymously.

Although growth monitoring had its principles defined first in less developed countries with "road to health" charts, it has been no less a feature of primary care in developed countries, with the traditional weighing scales being very much a part of health surveillance. As long ago as 1974, the Department of Health stated that "young babies should be weighed regularly so that assessment of growth by comparison with standard centile charts be made". ${ }^{1}$ In reality, growth monitoring is a screening procedure chiefly to diagnose undernutrition (for whatever reason). In the developed world it is also a tool to diagnose potential overnutrition. Garner et al scrutinised the world literature for randomised or quasi-randomised controlled clinical trials of weight, height or $\mathrm{mid} /$ upper arm circumference gain to seek evidence for benefit (or even harm) from growth monitoring. They were able to find only two trials that met their strict inclusion criteriaone from India and the other Lesotho and neither was able to show any real health benefits; findings that contrast with an almost evangelical conviction held by so many parents and health professionals that growth monitoring in less developed and developed countries is beneficial.

Should we really be surprised at these conclusions? The practice of medicine contains numerous examples of diagnostic and therapeutic interventions that are widely assumed to confer benefit but are supported by little real evidence. In 1983, Tom Williams and I appraised the then widely implemented clinical practice of weighing babies - asking if this procedure was worthwhile. We drew attention to poor weighing methods, cold rooms, variable amounts of clothes covering babies when weighed, use of inaccurate weighing scales, insufficient use of centile charts, and a poor understanding by those responsible for weighing babies of normal variations in weight profiles, including not inconsiderable shifts across centile lines. ${ }^{2}$ Our general conclusions were that, providing the measurement of weight was accurate, individual values plotted on centile charts, and the resulting profile sensibly interpreted, this could be a valuable method of health monitoring. But possibly the greatest benefit we felt was for mothers to be given the opportunity to discuss any queries 
about child care when bringing their babies to be weighed. Garner et al highlight the capacity of growth monitoring to cause harmespecially creating anxiety in the main carer, usually the mother, and the impact this might have on caregiving practices. Yet, in none of the studies they looked at had they found this matter to have been reliably addressed.

Over the years my views on growth monitoring have changed little-in spite of the introduction of more sophisticated growth charts and a widely established perception that growth monitoring is worthwhile. My outpatient clinic is frequently attended by infants referred because of abnormal weight gain, faltering or accelerating weight profiles, suspected following routine monitoring but not prompted by any particular clinical concern. It is also not unusual for babies to be referred with similarly suspected abnormal head growth. There is nothing wrong with the infants but the parents are invariably very anxious - a problem created out of nothing. I wonder whether the measurement of weight, and any other growth parameter, should be an investigation of possible abnormality rather than a routinely carried out primary clinical measurement, as - for example, the measurement of haemoglobin where anaemia is clinically suspected? My anecdotal worries receive support in Garner et al's paper. Perhaps a lot of good practice does go on where benefit is conferred but, if this is so, what a pity it is not published so that doubts can be dispelled. The fact that no controlled clinical trials seems to have been undertaken in developed countries only shows how deeply entrenched is this process of growth monitoring in our own clinical psyche.

What, therefore, the fate of growth surveillance if there is insufficient evidence for its benefits? Someone like myself working in hospital paediatric practice should not perhaps carry too much influence on what happens in primary care, especially for such an entrenched ritual as weighing babies; we must learn our lesson from similar mistakes in immunisation recommendations. However, I will stick my neck out! In the developing world I would hesitate to advise babies not to be routinely weighed. This must be a matter for those with intimate knowledge of localities where clinical practice takes place and, which, of course, will vary enormously in different parts of the world. In the developed world I have greater doubts about growth monitoring. Comments over many years from mothers who have derived little satisfaction from regular weighing, and who have sometimes suffered unnecessary worry, support my anxieties. Maybe there could be benefits from intelligent, regular weighing targeted to babies especially at risk from failure to thrive (including breast fed infants where lactation adequacy can sometimes be established only through regular careful weighing) but, again, we need more evidence. Maybe, as Garner et al indicate, an important spinoff of growth monitoring is the opportunity for mothers to ask questions about health issues, but this is only valuable if no harm or even complacency emerges from routine weighing, "primum non nocere".

However strong might be the academic argument against the clinical ritual of growth monitoring, I will have to concede (with some reluctance) that babies will continue to have their weight gain monitored. (It would take more than an Act of Parliament to stop it!) What, therefore, should we do to advance an existing unsatisfactory situation? Babies should be weighed on accurate weighing scales under constant environmental conditions with a minimum of clothes (preferably none), using appropriate centile charts with an understanding by those responsible how to interpret possible weight gain deviation. But there is also a need for further controlled clinical trials with clear definition of intervention and agreed outcomes (including medical referral and satisfaction indices) to establish the real value of this procedure. Why should the current renaissance of interest in the value of clinical effectiveness not apply to this most commonly used simple procedure. But I doubt somehow there will be much support for such studies. I wonder whether they would ever pass ethics committee scrutiny, so fixed are we in our biases. I will, however, continue to question the ethics of persisting with a clinical procedure, which is of unproved benefit, and with a capacity to do harm.

Professor of Child Health, University of Wales

D P DAVIES

College of Medicine, Heath Park, Cardiff CF14 4XN, UK

1 Department of Health and Social Security. Present day practice in infant feeding. London: HMSO, 1974:26.

2 Davies DP, Williams T. Is weighing babies in clinics worthwhile? BMf 1983;286:860-7. 